\title{
HIERARCHICAL HETEROGENITY OF POPULATIONS: MODELING BY THE OPEN EIGEN HYPERCYCLE
}

\author{
Vasiliy Ye. Belozyorov \\ Faculty of Applied Mathematics \\ O. Gonchara Dnipropetrovsk National University \\ 49010, Dnipropetrovsk, Ukraine \\ Email: belozvye@mail.ru
}

\author{
Serge V. Chernyshenko \\ Faculty of Informatics \\ University of Koblenz-Landau \\ D-56070, Koblenz, Germany \\ Email: svc@a-teleport.com
}

\author{
Vsevolod S. Chernyshenko \\ Computer Software Department \\ National Mining University \\ 49600, Dnipropetrovsk, Ukraine \\ Email: VseVsevolod@hotmail.com
}

\section{KEYWORDS}

System of ordinary quadratic differential equations, asymptotic stability, biological population.

\begin{abstract}
The case of a biological population, which consists of several sub-populations (different kinds of the population "social" groups: families, bevies, etc.), has been considered. For description of non-trivial interactions between these groups, a model of "open the Eigen hypercycle" has been proposed. Its bifurcation analysis for 3-dimension case has been carried out. Ecological interpretation of the results has been discussed.
\end{abstract}

\section{INTRODUCTION}

A huge number of mathematical models of ecological population structure (for example, (Allen 1974; Austin 1990; Billik and Case 1994; Chernyshenko 1995; Maurer 1999; Williamson 1990)) are designed for the description of population dynamics while taking into account different kinds of physiological differences between specimens (of age, sex, size, etc.) At the same time it is known (Breder 1959; Urich 1938) another form of population heterogeneity, based on the "social" population structure.Contrary to sex difference, specimens can change their group membership (though this changes are not as mechanistic as change of age). The main feature of social groups is an hierarchical character of the "social structure" and dependence of the existance of higher groups on the proper functionality of the lower ones. Big populations of relatively "intellectual" animals form new levels of population organization. The most bright example is the row "specimen - family - bevy" and, correspondingly, different kind of specimens (ordinary specimen, head of family, bevy leader) (Manteyfel 1992).
In the famous hypercycle model, proposed by Eigen and Shuster (Eigen and Schuster 1979; May 1991), similar relationship between system elements is described. At the same time, relations between "social groups" are not cyclic, and worsening of life conditions of the populations leads to elimination of higher levels while keeping lower ones. That gives an idea to use an open modification of the hypercycle model, which was proposed initially for the description of ecological successions and some social processes (Sole and Bascompte 2006; Ellner and Rees 2006).

The open hypercycle model is based on a matrix representation, which is non-linear (it is natural for a model of "social self-organization" of the population). In the article we focus on a continuous case, although the results may be used for discrete versions also. The model shows dependence of a complexity of population "social structure" from the size of its niche. Population "chooses" its complexity (or dimension) itself. We show it exactly for 3-dimension case (as well as for 2-dimension earlier (Chernyshenko 2005); generalization for $\mathrm{N}$-dimension case is matter of further research.

\section{OPEN HYPERCYCLE MODEL.}

Let's consider the dynamic behavior of the heterogeneous biological population $\mathbf{x}(t)=\left(x_{1}(t), \ldots, x_{n}(t)\right)^{T}$, which describing by Eigen's model hypercycle (here and further symbol $T$ signifies transposition)

$$
\left\{\begin{array}{l}
\dot{x}_{1}(t)=x_{1}(t)\left(F_{1}(t)-\frac{1}{S_{0}} \sum_{j=1}^{n} x_{j}(t) F_{j}(t)\right), \\
\ldots \ldots \ldots \ldots \ldots \\
\dot{x}_{n}(t)=x_{n}(t)\left(F_{n}(t)-\frac{1}{S_{0}} \sum_{j=1}^{n} x_{j}(t) F_{j}(t)\right),
\end{array}\right.
$$


(here $S_{0}>0$ ). The population consists of $n$ subpopulations $x_{i}(t), i=1, \ldots, n$, which represent different levels of "social" hierarchy. The vector of initial values is $\mathbf{x}^{T}(0)=\left(x_{10}, \ldots, x_{n 0}\right)$.

We will consider that $F_{1}(t)=N-x_{1}(t), F_{i}(t)=$ $a_{i-1} x_{i-1}(t)-x_{i}(t) ; i=2, \ldots, n$, where $N, a_{1}, \ldots, a_{n-1}$ are positive numbers. (The functions $F_{1}(t), \ldots, F_{n}(t)$ are called as Allen's functions (Allen 1974).)

These functions determine very special interaction between the sub-populations, whene each of them depends on all the previous ones. Contrary to Eigen's hypercycle, the dependence has no cyclic character, so we can call this model as 'open' hypercycle.

\section{THE EQUILIBRIUM POINTS OF THE SYSTEM}

(1).

Let's investigate analytically main features of the model dynamics.

Let's define as $i_{1}, \ldots, i_{k}(k \leq n)$ permutations of any $k$ symbols $1,2, \ldots, n$, for which the condition

$$
1 \leq i_{1}<\ldots<i_{k} \leq n
$$

is fulfilled.

Assume that $x_{i_{1}} \neq 0, \ldots, x_{i_{k}} \neq 0$ and $x_{i_{k+1}}=$ $0, \ldots, x_{i_{n}}=0$. Then the system of equations that define equilibriums has the form

$$
\begin{gathered}
\left(\begin{array}{cccc}
1-\frac{x_{i_{1}}}{S_{0}} & -\frac{x_{i_{2}}}{S_{0}} & \ldots & -\frac{x_{i_{k}}}{S_{0}} \\
-\frac{x_{i_{1}}}{S_{0}} & 1-\frac{x_{i_{2}}}{S_{0}} & \ldots & -\frac{x_{i_{k}}}{S_{0}} \\
\vdots & \vdots & \ddots & \vdots \\
-\frac{x_{i_{1}}}{S_{0}} & -\frac{x_{i_{2}}}{S_{0}} & \cdots & 1-\frac{x_{i_{k}}}{S_{0}}
\end{array}\right) \cdot\left(\begin{array}{c}
F_{i_{1}} \\
F_{i_{2}} \\
\vdots \\
F_{i_{k}}
\end{array}\right)=0 \\
x_{i_{k+1}}=\ldots=x_{i_{n}}=0
\end{gathered}
$$

A determinant of the first $k$ equations of system (2) may be presented as:

$$
\operatorname{det}\left(\begin{array}{cccc}
1-\frac{x_{i_{1}}}{S_{0}} & -\frac{x_{i_{2}}}{S_{0}} & \cdots & -\frac{x_{i_{k}}}{S_{0}} \\
-\frac{x_{i_{1}}}{S_{0}} & 1-\frac{x_{i_{2}}}{S_{0}} & \cdots & -\frac{x_{i_{k}}}{S_{0}} \\
\vdots & \vdots & \ddots & \vdots \\
-\frac{x_{i_{1}}}{S_{0}} & -\frac{x_{i_{2}}}{S_{0}} & \cdots & 1-\frac{x_{i_{k}}}{S_{0}}
\end{array}\right)=
$$

Here we may face two cases: 1$) 1-\frac{x_{i_{1}}+\ldots+x_{i_{k}}}{S_{0}} \neq 0$ and 2) $1-\frac{x_{i_{1}}+\ldots+x_{i_{k}}}{S_{0}}=0$.
2.1. Determinant of the first $k$ equations of the system (1) is not equal to zero.

In this case, according to (2) we get that $F_{i_{1}}=\ldots=F_{i_{k}}=$ $x_{i_{k+1}}=\ldots=x_{i_{n}}=0$. Condition $F_{i_{1}}=0$ leads to $x_{1} \neq 0$, then $x_{1}=N, x_{2}=a_{1} N, x_{3}=a_{1} a_{2} N, \ldots, x_{n}=a_{1} \cdot \ldots$. $a_{n-1} N$; in this case the condition

$$
S_{0} \neq N\left(1+a_{1}+a_{1} a_{2}+\ldots+a_{1} \cdot \ldots \cdot a_{n-1}\right)
$$

has to be fulfilled.

Let $x_{1} \neq 0, x_{2}=0$. Then $x_{3}=\ldots=x_{n}=0$. Assume $x_{1} \neq 0, F_{2}=0$. Then $x_{1}=N, x_{2}=a_{1} N, x_{3}=\ldots=$ $x_{n}=0$.

It is easy to find out that in this case we have $n+1$ equilibriums:

$$
E_{1}=\left(\begin{array}{c}
0 \\
0 \\
\vdots \\
0
\end{array}\right), \ldots, E_{k+1}=\left(\begin{array}{c}
1 \\
a_{1} \\
\vdots \\
a_{1} \cdot \ldots \cdot a_{k-1} \\
0 \\
\vdots \\
0
\end{array}\right) N, \ldots
$$

$$
E_{n+1}=\left(\begin{array}{c}
1 \\
a_{1} \\
\vdots \\
a_{1} \cdot \ldots \cdot a_{n-1}
\end{array}\right) N \in \mathbb{R}^{n} .
$$

\subsection{Determinant of the first $k$ equations of the system} (1) is equal to zero.

Here, we have $x_{i_{k}}=S_{0}-x_{i_{1}}-\ldots-x_{i_{k-1}}$. Substituting the last formula in the system (2) we derive $F_{i_{1}}=F_{i_{2}}=$ $\ldots=F_{i_{k}}=F$, where $F$ is a nonzero function. Taking into account the last equations, system (2) may be presented as

$$
\left\{\begin{array}{l}
x_{i_{1}}\left(a_{i_{1}-1} x_{i_{1}-1}-x_{i_{1}}-F\right)=0, \\
\cdots \cdots \\
x_{i_{k}}\left(a_{i_{k}-1} x_{i_{k}-1}-x_{i_{k}}-F\right)=0, \\
x_{1}+\ldots+x_{n}=S_{0}, \\
x_{i_{k+1}}=0, \\
\cdots \cdots \\
x_{i_{n}}=0,
\end{array}\right.
$$

where $F \neq F_{i_{k+1}}, \ldots, F \neq F_{i_{n}}$.

From this system we derive that $x_{i_{1}} \neq 0, \ldots, x_{i_{k}} \neq 0$. As $k=1,2, \ldots, n-1$, we get $C_{n}^{1}+C_{n}^{2}+\ldots+C_{n}^{n-1}+C_{n}^{n}$ solutions. Here $C_{n}^{i}$ is a number of combinations of $k$ sets from $n$ elements $(k<n)$.

Taking into account the case when determinant of the rst $\mathrm{k}$ equations of the system (1) is not equal to zero, we get for this system (1) $n+1+C_{n}^{1}+\ldots+C_{n}^{n-1}+1=2^{n}+n$ equilibriums. 


\subsection{Jacobian matrix building.}

The Jacobi matrix for arbitrary $n$ can be evaluated as $J=$ $A+B+C$, where:

$$
\begin{aligned}
& A=\left(\begin{array}{ccc}
F_{1}-\frac{1}{S_{0}} \sum_{j=1}^{n} x_{j} F_{j} & \cdots & 0 \\
\vdots & \ddots & \vdots \\
0 & \cdots & F_{n}-\frac{1}{S_{0}} \sum_{j=1}^{n} x_{j} F_{j}
\end{array}\right) \\
& B=\left(\begin{array}{ccccc}
-x_{1} & 0 & 0 & \cdots & 0 \\
x_{2} a_{1} & -x_{2} & 0 & \cdots & 0 \\
\vdots & \vdots & \ddots & \ddots & \vdots \\
0 & 0 & \cdots & x_{n} a_{n-1} & -x_{n}
\end{array}\right)
\end{aligned}
$$

$C=-\frac{1}{S_{0}}\left(\begin{array}{ccc}x_{1}\left(N-2 x_{1}+a_{1} x_{2}\right) & \cdots & x_{1}\left(a_{n-1} x_{n-1}-2 x_{n}\right) \\ \vdots & \cdots & \vdots \\ x_{n}\left(N-2 x_{1}+a_{1} x_{2}\right) & \cdots & x_{n}\left(a_{n-1} x_{n-1}-2 x_{n}\right)\end{array}\right)$.

\section{EIGENVALUES OF JACOBI MATRIX IN EQUI- LIBRIUM POINTS FOR $\mathbf{n}=\mathbf{3}$.}

Let $n=3$ and $F_{1}(t)=N-x_{1}(t), F_{2}(t)=a_{1} x_{1}(t)-$ $x_{2}(t), F_{3}(t)=a_{2} x_{2}(t)-x_{3}(t)$, where $N>0, a_{1}>$ $0, a_{2}>0$.

Here we come to two cases: 1$) x_{1}+x_{2}+x_{3} \neq S_{0}$ and 2) $x_{1}+x_{2}+x_{3}=S_{0}$.

In the first case $\left(x_{1}+x_{2}+x_{3} \neq S_{0}\right)$ we have four equilibriums:

$$
(0,0,0)^{T},(N, 0,0)^{T},\left(N, a_{1} N, 0\right)^{T},\left(N, a_{1} N, a_{1} a_{2} N\right)^{T} .
$$

In the second case $\left(x_{1}+x_{2}+x_{3}=S_{0}\right)$ we have seven systems of equations, watch (3), to determine additional seven equilibriums:

$$
\begin{gathered}
\left\{\begin{array}{l}
a_{1} x_{1}-x_{2}-F=0 \\
a_{2} x_{2}-x_{3}-F=0 \\
x_{1}+x_{2}+x_{3}=S_{0} \\
x_{1}=0
\end{array},\left\{\begin{array}{l}
N-x_{1}-F=0 \\
a_{2} x_{2}-x_{3}-F=0 \\
x_{1}+x_{2}+x_{3}=S_{0} \\
x_{2}=0
\end{array},\right.\right. \\
\left\{\begin{array}{l}
N-x_{1}-F=0 \\
a_{1} x_{1}-x_{2}-F=0 \\
x_{1}+x_{2}+x_{3}=S_{0} \\
x_{3}=0
\end{array},\left\{\begin{array}{l}
a_{2} x_{2}-x_{3}-F=0 \\
x_{1}+x_{2}+x_{3}=S_{0} \\
x_{1}=0 \\
x_{2}=0
\end{array},\right.\right. \\
\left\{\begin{array}{l}
N-x_{1}-F=0 \\
x_{1}+x_{2}+x_{3}=S_{0} \\
x_{2}=0 \\
x_{3}=0
\end{array},\left\{\begin{array}{l}
a_{1} x_{1}-x_{2}-F=0 \\
x_{1}+x_{2}+x_{3}=S_{0} \\
x_{1}=0 \\
x_{3}=0
\end{array},\right.\right. \\
\left\{\begin{array}{l}
N-x_{1}-F=0 \\
a_{1} x_{1}-x_{2}-F=0 \\
a_{2} x_{2}-x_{3}-F=0 \\
x_{1}+x_{2}+x_{3}=S_{0}
\end{array}\right.
\end{gathered}
$$

(Indefinite form of function $F$ doesn't affect the present analysis.)

So, for the system (1) where $n=3$ we got following 11 equilibriums:

$E_{1}$ :

$$
x_{1}=0, x_{2}=0, x_{3}=0 \text {. }
$$

$E_{2}:$

$$
x_{1}=0, x_{2}=\frac{S_{0}}{a_{2}+2}, x_{3}=\frac{\left(a_{2}+1\right) S_{0}}{a_{2}+2} .
$$

$E_{3}:$

$$
x_{1}=\frac{S_{0}+N}{2}, x_{2}=0, x_{3}=\frac{S_{0}-N}{2} .
$$

$E_{4}:$

$$
x_{1}=\frac{S_{0}+N}{a_{1}+2}, x_{2}=\frac{\left(a_{1}+1\right) S_{0}-N}{a_{1}+2}, x_{3}=0 .
$$

$E_{5}:$

$$
x_{1}=N, x_{2}=a_{1} N, x_{3}=0 .
$$

$E_{6}:$

$$
x_{1}=0, x_{2}=0, x_{3}=S_{0}
$$

$E_{7}:$

$$
x_{1}=0, x_{2}=S_{0}, x_{3}=0 .
$$

$E_{8}:$

$$
x_{1}=S_{0}, x_{2}=0, x_{3}=0 .
$$

$E_{9}:$

$$
x_{1}=N, x_{2}=0, x_{3}=0 \text {. }
$$

$E_{10}:$

$$
F_{1}=F_{2}=F_{3}=0
$$

in this case

$$
x_{1}=N, x_{2}=a_{1} N, x_{3}=a_{1} a_{2} N .
$$

$E_{11}:$

$$
F_{1}=F_{2}=F_{3} \neq 0, x_{1}+x_{2}+x_{3}=S_{0}
$$

then

$$
\begin{gathered}
x_{1}=\frac{S_{0}+\left(a_{2}+2\right) N}{3+a_{1}+a_{2}+a_{1} a_{2}}, x_{2}=\frac{\left(a_{1}+1\right) S_{0}+\left(a_{1}-1\right) N}{3+a_{1}+a_{2}+a_{1} a_{2}}, \\
x_{3}=\frac{\left(1+a_{2}+a_{1} a_{2}\right) S_{0}-\left(a_{1}+a_{2}+1\right) N}{3+a_{1}+a_{2}+a_{1} a_{2}} .
\end{gathered}
$$

(It is easy to find that all equilibriums do exist in the first orthan, necessary and sufficient criteria are: $S_{0} \geq N$ and $a_{2} \geq 1$ will be fulfilled.) 
Now we are going to find a positive invariant set of the system (1) in case $n=3$. Sum of all equations of system (1) is equal to:

$$
\begin{gathered}
\frac{d\left(x_{1}+x_{2}+x_{3}-S_{0}\right)}{d t}=\left(x_{1}+x_{2}+x_{3}-S_{0}\right) * \\
* \frac{1}{S_{0}}\left(-N x_{1}-a_{1} x_{1} x_{2}-a_{2} x_{2} x_{3}+x_{1}^{2}+x_{2}^{2}+x_{3}^{2}\right) .
\end{gathered}
$$

The behavior of solutions of (7) is described by the matrix

$$
\left(\begin{array}{ccc}
1 & -a_{1} / 2 & 0 \\
-a_{1} / 2 & 1 & -a_{2} / 2 \\
0 & -a_{2} / 2 & 1
\end{array}\right)
$$

As $x_{1} \geq 0, x_{2} \geq 0, x_{2} \geq 0$, then it follows from the equation (7) that if $x_{10}+x_{20}+x_{30} \leq S_{0}$, in case $t \geq 0$, $x_{1}+x_{2}+x_{3} \leq S_{0}$. Let's define by $V$ a domain in the first orthant bounded by coordinate planes $x_{1}=0, x_{2}=0$, $x_{2}=0$ and $x_{1}+x_{2}+x_{3}=S_{0}$.

Recasting the equation (7) by successively completing the squares, we obtain:

$$
\begin{gathered}
\frac{d\left(x_{1}+x_{2}+x_{3}-S_{0}\right)}{d t}= \\
=\left(x_{1}+x_{2}+x_{3}-S_{0}\right) \frac{1}{S_{0}}\left(-N x_{1}+\left(x_{1}-0.5 a_{1} x_{2}\right)^{2}\right. \\
\left.+\left(x_{3}-0.5 a_{2} x_{2}\right)^{2}+\left(1-0.25 a_{1}^{2}-0.25 a_{2}^{2}\right) x_{2}^{2}\right)= \\
=\left(x_{1}+x_{2}+x_{3}-S_{0}\right) \frac{1}{S_{0}}\left[\left(x_{1}-\frac{a_{1}}{2} x_{2}-\frac{N}{2}\right)^{2}+\right. \\
+\frac{4-a_{1}^{2}-a_{2}^{2}}{4}\left(x_{2}-\frac{2 a_{1} N}{4-a_{1}^{2}-a_{2}^{2}}\right)^{2}+\left(x_{3}-\frac{a_{2}}{2} x_{2}\right)^{2}- \\
\left.-\frac{N^{2}}{4}-\frac{a_{1}^{2} N^{2}}{4-a_{1}^{2}-a_{2}^{2}}\right] .
\end{gathered}
$$

Let $a_{1}^{2}+a_{2}^{2}<4$. Define by $W$ an ellipsoid with center in point

$$
K\left(\frac{N}{2}+\frac{a_{1}^{2} N}{4-a_{1}^{2}-a_{2}^{2}}, \frac{2 a_{1} N}{4-a_{1}^{2}-a_{2}^{2}}, \frac{a_{1} a_{2} N}{4-a_{1}^{2}-a_{2}^{2}}\right)
$$

and product of semiaxes

$$
\frac{N^{3}\left(4+3 a_{1}^{2}-a_{2}^{2}\right)^{3 / 2}}{4\left(4-a_{1}^{2}-a_{2}^{2}\right)^{2}}
$$

If $a_{1}^{2}+a_{2}^{2}<4, x_{10}+x_{20}+x_{30} \geq S_{0}$ and $\left(x_{10}, x_{20}, x_{30}\right)^{T} \in W$, then a vector $\left(x_{1}(t), x_{2}(t), x_{3}(t)\right)^{T} \in W, \quad$ and the formula in big rectangular brackets is negative. Therefore $\lim _{t \rightarrow \infty}\left(x_{1}(t), x_{2}(t), x_{3}(t)\right)^{T}=E_{11}$. (Let $u=x_{1}(t)+x_{2}(t)+x_{3}(t)-S_{0}$. Then the equation (7) may be written in the form $\dot{u}=\xi(u) u$, where $\xi(u)<0$; we derive the analog of a linear equation, the solution of which tends to zero.) Further we will consider the case $\left(x_{10}, x_{20}, x_{30}\right)^{T} \in V$ only.

\section{ANALYSIS OF THE EQUILIBRIUMS.}

In all computations elements of Jacobian matrix divided on $S_{0}$ (so eigenvalue $\lambda$ is replaced by the construction $\lambda / S_{0}$ ).

Let $\mu=N / S_{0}$. This value characterizes the ecological niche's size (it is inversely proportional the size). Let's analyse a behavior of the system (1) in the vicinity of all equilibriums.

Let's estimate all eigenvalues:

$E_{1}$. In this point eigenvalues $\lambda_{1}=\mu, \lambda_{2}=\lambda_{3}=0$. Equilibrium is a degenerative unstable node.

$E_{2}$. In this point eigenvalues

$$
\lambda_{1}=\mu+\frac{1}{a_{2}+2}, \lambda_{2}=-\frac{a_{2}+1}{a_{2}+2}, \lambda_{3}=\frac{1}{a_{2}+2} .
$$

As $\mu>0, a_{2} \geq 0$ and $\lambda_{2} \leq 0$, equilibrium is a saddlenode.

$$
E_{3} \text {. Here }
$$

$$
\lambda_{1}=\frac{\mu\left(a_{1}-1\right)}{2}+\frac{a_{1}+1}{2}, \lambda_{2}=-\frac{1-\mu^{2}}{2}, \lambda_{3}=\frac{1-\mu}{2} .
$$

If $\mu \neq 1$, then from condition $\lambda_{2} \lambda_{3}<0$ it follows that this point is saddle-node. If $\mu=1$, then

$$
\lambda_{1}=a_{1}, \lambda_{2}=\lambda_{3}=0
$$

and we get a degenerative unstable node.

$E_{4}$. Here

$$
\begin{gathered}
\lambda_{1}=-\frac{\mu\left(1+a_{1}+a_{2}\right)}{a_{1}+2}+\frac{1+a_{2}+a_{1} a_{2}}{a_{1}+2}, \\
\lambda_{2}=-\frac{\mu\left(1+a_{1}\right)}{a_{1}+2}+\frac{1}{a_{1}+2}, \lambda_{3}=\frac{(\mu+1)\left(\mu-1-a_{1}\right)}{a_{1}+2} .
\end{gathered}
$$

A simple analysis shows that if

$$
\frac{1+a_{2}+a_{1} a_{2}}{1+a_{1}+a_{2}}<\mu<1+a_{1},
$$

then this point is a stable node. If $\mu>1+a_{1}$, then it is a saddle-node.

$E_{5}$. Here

$$
\begin{gathered}
\lambda_{1}=a_{2} a_{1} \mu, \lambda_{2,3}=-\frac{\mu\left(a_{1}+1-\mu\right)}{2} \pm \\
\pm \frac{\mu}{2} \sqrt{\left(a_{1}+1-\mu\right)^{2}+4 a_{1}\left(a_{1} \mu+\mu-1\right)} .
\end{gathered}
$$

As $\lambda_{1}>0$, then equilibrium is either a saddle or an unstable node.

$E_{6}$. Here $\lambda_{1}=1+\mu, \lambda_{2,3}=1$. Equilibrium is an unstable node.

$E_{7}$. Here $\lambda_{1}=1+\mu, \lambda_{2}=1, \lambda_{3}=a_{2}+1$. Equilibrium is an unstable node.

$E_{8}$. Here $\lambda_{1,2}=1-\mu, \lambda_{3}=a_{1}+1-\mu$. This point is either an unstable node or a stable node or a saddle-node. 
$E_{9}$. Here $\lambda_{1}=\mu(\mu-1), \lambda_{2}=a_{1} \mu, \lambda_{3}=0$. This point is an unstable node or saddle.

$E_{10}$. It is clear that $N\left(1+a_{1}+a_{1} a_{2}\right) \leq S_{0}$ or

$$
\mu=\frac{S_{0}}{N} \leq \frac{1}{1+a_{1}+a_{1} a_{2}}
$$

then $E_{10} \subset V$.

The Jacobi matrix in point $E_{10}$ may be presented as:

$$
\begin{gathered}
J_{10} / S_{0}=\mu\left(\begin{array}{ccc}
-1 & 0 & 0 \\
a_{1}^{2} & -a_{1} & 0 \\
0 & a_{1} a_{2}^{2} & -a_{1} a_{2}
\end{array}\right)+ \\
+\mu^{2}\left[\left(\begin{array}{c}
1 \\
a_{1} \\
a_{1} a_{2}
\end{array}\right) \cdot\left(\left(1-a_{1}^{2}\right), a_{1}\left(1-a_{2}^{2}\right), a_{1} a_{2}\right)\right] .
\end{gathered}
$$

A characteristic polynomial of the Jacobi matrix in point $E_{10}$ is:

$$
\begin{gathered}
\operatorname{det}\left(\lambda I-J_{10} / S_{0}\right)=\lambda^{3}+p_{1} \lambda^{2}+p_{2} \lambda+p_{3}= \\
=\lambda^{3}+\left[\mu\left(1+a_{1}+a_{1} a_{2}\right)-\mu^{2}\right] \lambda^{2}+\left[a_{1}\left(1+a_{2}+a_{1} a_{2}\right) \mu^{2}-\right. \\
\left.-a_{1}\left(1+a_{1}+a_{2}\right) \mu^{3}\right] \lambda+a_{1}^{2} a_{2}\left[\mu^{3}-\left(1+a_{1}+a_{1} a_{2}\right) \mu^{4}\right] .
\end{gathered}
$$

Let's assume that the condition (6) is satisfied. Then $E_{10} \subset V$. If $\mu \rightarrow 0$, then this point is asymptotic stable. In case when $\mu$ is increasing, but inequality (6) is satisfied, it easy to check that $p_{1}>0, p_{2}>0, p_{3}>0, p_{1} p_{2}>p_{3}$. If $\mu=\left(1+a_{1}+a_{1} a_{2}\right)^{-1}$ (bifurcation point), then $\lambda_{1}=0$ and the point turns out to be unstable.

$E_{11}$. One of eigenvalues of the Jacobi matrix is

$$
\lambda_{1}=\frac{1-\mu\left(1+a_{1}+a_{1} a_{2}\right)}{3+a_{1}+a_{2}+a_{1} a_{2}} .
$$

Therefore the inequality (6) is hold. So $\lambda_{1}>0$ and equilibrium $E_{11}$ may be node or saddle.

If

$$
\mu \approx \frac{1}{1+a_{1}+a_{1} a_{2}}
$$

and

$$
\mu>\frac{1}{1+a_{1}+a_{1} a_{2}},
$$

then equilibrium is a stable node. Thus, if

$$
\mu=\frac{1}{1+a_{1}+a_{1} a_{2}}
$$

then points $E_{10}$ and $E_{11}$ are exchange.

Consider the case

$$
\mu>\frac{1}{1+a_{1}+a_{1} a_{2}} .
$$

It is clear that

$$
\frac{1}{1+a_{1}+a_{1} a_{2}} \leq \frac{1+a_{2}+a_{1} a_{2}}{1+a_{1}+a_{2}} .
$$

Note, if

$$
\mu=\frac{1}{1+a_{1}+a_{1} a_{2}}
$$

then one of eigenvalues of the Jacobi matrix in the point $E_{11}$ equal to zero; if

$$
\mu=\frac{1+a_{2}+a_{1} a_{2}}{1+a_{1}+a_{2}},
$$

then another eigenvalue of the Jacobi matrix equal to zero (other two eigenvalues have negative real parts).

If

$$
\frac{1}{1+a_{1}+a_{1} a_{2}}<\mu<\frac{1+a_{2}+a_{1} a_{2}}{1+a_{1}+a_{2}},
$$

then the point $E_{11}$ is stable; if

$$
\mu>\frac{1+a_{2}+a_{1} a_{2}}{1+a_{1}+a_{2}},
$$

then the point $E_{11}$ is unstable.

At last, if the condition (5) is fulfilled, then we have the stable equilibrium in $E_{4}$.

\section{BIFURCATION POINTS.}

Let's find a positive invariant set of the system (1) for $n=$ 3. Add all equations of system (1):

$$
\begin{aligned}
& \frac{d\left(x_{1}+x_{2}+x_{3}-S_{0}\right)}{d t}=\left(x_{1}+x_{2}+x_{3}-S_{0}\right) \frac{1}{S_{0}}\left(-N x_{1}-\right. \\
& \left.-\mathrm{a}_{1} x_{1} x_{2}-a_{2} x_{2} x_{3}+x_{1}^{2}+x_{2}^{2}+x_{3}^{2}\right) .(7)
\end{aligned}
$$

Let's introduce a new variable $u(t)=x_{1}(t)+x_{2}(t)+$ $x_{3}(t)-S_{0}$. Then equation (??) may be written as

$$
\frac{d u}{d t}=u \frac{1}{S_{0}}\left(-N x_{1}-a_{1} x_{1} x_{2}-a_{2} x_{2} x_{3}+x_{1}^{2}+x_{2}^{2}+x_{3}^{2}\right) .
$$

Theorem 1. Any solution $u(t)$ (for any initial value $u_{0}=u(0)$ and $\left.\forall t \geq 0\right)$ of equation (8) has the property: $u_{0} u(t) \geq 0$.

Let $u_{0} \leq 0$. As $x_{1} \geq 0, x_{2} \geq 0, x_{2} \geq 0$, then according to the Theorem 1 while $t \geq 0$, the function $u(t)=x_{1}(t)+$ $x_{2}(t)+x_{3}(t)-S_{0} \leq 0$. We will define as $V$ a domain in the first orthant, bounded by coordinate planes $x_{1}=0$, $x_{2}=0, x_{2}=0$ and $x_{1}+x_{2}+x_{3}=S_{0}$. Then, we derive that $V$ is an invariant set $\left(\forall t \geq 0\right.$ from $x_{10}+x_{20}+x_{30} \leq S_{0}$ it follows that $\left.x_{1}(t)+x_{2}(t)+x_{3}(t) \leq S_{0}\right)$.

We will consider that $\left(x_{10}, x_{20}, x_{30}\right)^{T} \in V$ is a vector of initial values. In this case we will have $\forall t>0$ $\left(x_{1}(t), x_{2}(t), x_{3}(t)\right)^{T} \in V$. Thus, $V$ is a positive invariant set.

We suppose that $x_{10}>0, x_{20}>0, x_{30}>0$. (If one initial value is zero, then we will come to a $2-\mathrm{D}$ analysis.) 
There are 4 different bifurcations points:

$$
\begin{gathered}
\mu_{0}=0, \mu_{1}=\frac{1}{1+a_{1}+a_{1} a_{2}}, \mu_{2}=\frac{1+a_{2}+a_{1} a_{2}}{1+a_{1}+a_{2}}, \\
\mu_{3}=1+a_{1} .
\end{gathered}
$$

1.

$$
0<\mu<\frac{1}{1+a_{1}+a_{1} a_{2}} .
$$

The trajectory tends to the single equilibrium $E_{10} \in V$. Thus, for small $\mu$ (when the size of the ecological niche larger then the critical value: $\left.S_{0}>\left(1+a_{1}+a_{1} a_{2}\right) \cdot N\right)$, all sub-populations do exist and try to reach maximal possible size; on the other hand, their aggregate size is smaller then the size $S_{0}$ of the population niche.

2 .

$$
\frac{1}{1+a_{1}+a_{1} a_{2}}<\mu<\frac{1+a_{2}+a_{1} a_{2}}{1+a_{1}+a_{2}} .
$$

The trajectory tends to the single equilibriums $E_{11} \in V$. With such a size of the ecological niche, all three subpopulations may be presented; they occupy whole ecological niche $S_{0}$ and are limited in size by the size of the niche.

3.

$$
\frac{1+a_{2}+a_{1} a_{2}}{1+a_{1}+a_{2}}<\mu<1+a_{1} .
$$

The trajectory tends to the single equilibriums $E_{4} \in V$. The niche size $S_{0}$ is so small that the third level of the subpopulation "social" organization (the third sub-population) can not come into existence. Two other populations occupy the entire ecological niche.

4. $1+a_{1}<\mu<\infty$.

In this case the trajectory tends to the point $E_{8} \in V$. In the environment of such a small ecological niche only the first subpopulation is able to exist. So population is limited only by the size of niche. It has no internal structure; second and third sub-populations are absent.

\section{CONCLUSION.}

The analysis of results which were resulted above, leads to the several observations. In case of small values of $p$, only the first subpopulation survives. Second and third subpopulations survive just with growth of $p$. In addition, all surviving populations occupy whole ecological niche. Finally, if $p>p_{3}$ then an "era of abundance" comes, when all populations survive, and ecological niche is filled partially (all resources of the niche are used not completely).

One should also mention the following: parameters $S_{0}, N, a_{1}, a_{2}$ have different influence on a character of flowing processes. The most important of these parameters are $S_{0}, N$. Parameter $S_{0}$ determines the maximal volume of resources, that can be used while populations development. Parameter $N$ shows that at any nonzero $S_{0}$ the first population always survives. This conclusion allows to assert that in case of the Eygen model, the biological association remains (what is possible thanks to the reduced structure). Parameters $a_{1} a n d a_{2}$ specify only a quantitative influence of one population on others. In addition, these parameters do not change the type of bifurcation points and their ammount.

Analysis of the 3-D open Eigen's model allows to propose the following suggestion sfor $n$-dimension case.

There do exist $n+1$ bifurcation points :

$$
0=\mu_{0}<\mu_{1}<\mu_{2}<\ldots<\mu_{n}<\infty,
$$

where

$$
\mu_{1}=\frac{1}{1+a_{1}+a_{1} a_{2}+\ldots+a_{1} \cdot \ldots \cdot a_{n-1}} .
$$

If

$$
0<\mu<\mu_{1},
$$

then all populations survive and occupy only part of ecological niche; a volume of this niche is $N\left(1+a_{1}+a_{1} a_{2}+\right.$ $\left.\ldots+a_{1} \cdot \ldots \cdot a_{n-1}\right)<S_{0}$.

If:

$$
\mu_{1} \leq \mu<\infty,
$$

then populations (but not all) occupy whole ecological niche $S_{0}$; if

$$
\mu_{i}<\mu<\mu_{i+1}, 1 \leq i \leq n-1,
$$

then populations $1,2, \ldots, n-i+1$ remain and populations $n-1, n-2, \ldots, i-1$ die out ; when

$$
\mu_{n}<\mu<\infty
$$

then only unique population survive and fill whole ecological niche $S_{0}$.

\section{REFERENCES}

[Ackleh and Allen 2003] Ackleh A. S., Allen L. J. S. 2003. Competitive exclusion and coexistence for pathogens in an epidemic model with variable population size. J. Math. Biol. Vol.47, 153-168.

[Allen 1974] Allen P. M. 1974. ”Evolution, population dynamics and stability". Proc. of the National Academy of Sciences of the USA. Vol. 73, No 3, 665-668.

[Austin 1990] Austin M. P. 1990. Community theory and competition in vegetation. Perspectives on plant competition. San Diego: Academic Press, 215-238.

[Billik and Case 1994] Billik I., Case T. J. 1994. Higher order interactions in ecological communities: What are they and how can they be detected. . Ecology. Vol. 75, 1529-1543.

[Breder 1959] Breder C. M. 1959. Studies on social grouping in fishes. -Bull. Amer. Mus. Natur. Hist. Vol. 117. No 6, 397-481. 
[Caswell 2001] Caswell H. 2001. Matrix population models:Constructions, Analisis, and Inerpretation. 2nd. ed. Sinauer. Sunderland MA.

[Chernousenko et al. 1988] Chernousenko V. M., Chernenko I. V., Chernyshenko S. V. 1988. Bifurcation in the modified Eigen hypercycle. Nonlinear and turbulent processes in physics. Proc. of 3rd Intern. workshop. Kiev: Naukova Dumka. Vol. 2, 261-263.

[Chernyshenko 1995] Chernyshenko S. V. 1995. The open Eigen Hypercycle and selforganization of ecological systems. Sustainable Development: Environmental Pollution and Ecological Safety. Dniepropetrovsk: Dniepropetrovsk Univ. Press, 42-43.

[Chernyshenko 2005] Chernyshenko S.V. 2005. Nonlinear analysis of forest ecosystems dynamics. Dnipropetrovsk, Dnipropetrovsk University Press.

[Deng 2008] Deng B. 2008. The time invariance principle, the absence of ecological chaos, and a fundamental pitfall of discrete modeling. Ecologicall Modelling. Vol.215, 287292.

[Easterling et al. 2000] Easterling M. R.,Ellner S. P., Dixon P. 2000. Size-specific sensitivity: applying a new structured population model. Ecology. Vol.81, 964-708.

[Eigen and Schuster 1979] Eigen M., Schuster P. 1979. The hypercycle: A principle of natural selforganization. Spriger: Berlin.

[Ellner and Rees 2006] Ellner S. P., Rees M. 2006. Integral projection models for species with complex demography. American Naturalist. Vol.167, 410-428.

[Levin 1997] Levin S. A. 1997. Models of population dispersal. Differential Equations and Applications in Ecology, Epidemics, and Population Problems. New-York: L. Academic Press, 1-18.

[Manteyfel 1992] Manteyfel B. P. 1992. Ecological and evolutional aspects of animal behaviour. Moscow: Nauka.

[Maurer 1999] Maurer B. A. 1999. Untangling ecological complexity. Chicago : Univ. of Chicago Press.

[May 1991] May R.M. 1991. Hypercycles spring to life. Nature. Vol.353, 607-609.

[Odum 1994] Odum H. T. 1994. Ecological and general systems and introduction to systems ecology. Niwot: University Press of Colorado.

[Roff 1974] Roff D. A. 1974. Spatial heterogenity and persistence of populations. Ecologia. Vol. 15, 245-258.

[Sole and Bascompte 2006] Sole R.V., Bascompte J. 2006. SelfOrganization in Complex Ecosystems. Monograps in Population Biology. Prinston University Press. Vol.42.
[Urich 1938] Urich I. 1938. The social hierarchy in albino mice. J. Comp. Psychol. Vol. 25., 373-413.

[Williamson 1990] Williamson M. 1990. The analysis of biological populations. L.: Academic Press.

\section{AUTHOR BIOGRAPHIES}

VASILIY Ye. BELOZYOROV was born in Dnipropetrovsk, Ukraine graduated from the Dnipropetrovsk State University. Obtained $\mathrm{PhD}$ degree on mathematical system theory in 1986. From 1996 till 2009 was, successively, head of Applied Mathematics Department at Dnipropetrovsk National University. $\mathrm{He}$ is an Honoured Science Worker of Ukraine. In 2010 he became a professor of Computer Technologies Department, Dnipropetrovsk National University. His e-mail address is belozvye@mail.ru

SERGE V. CHERNYSHENKO was born in Dnipropetrovsk, Ukraine and graduated from the Dnipropetrovsk State Uni-versity. After obtaining PhD degree on computing in 1986, was, successively, head of Laboratory of Mathematical Modeling in Biology, head of Computer Science Department, dean of Applied Mathematics Faculty at Dnipropetrovsk National University. He was a supervisor of several Ukrainian national projects on mathematical modeling. From 2009 he is a visiting professor of KoblenzLandau University, Faculty of Informatics. His e-mail address is svcla-teleport.com and his web-site can be found at www. uni-koblenz.de/ svc.

VSEVOLOD S. CHERNYSHENKO was born in Dnipropetrovsk, Ukraine and graduated from the Dnipropetrovsk National University. Got PhD degree on Mathematical Modeling and Numerical Methods in 2009. Nowadays he is a docent at the Computer Software Department of the National Mining University of Ukraine. He is a member of editorial board of journals "Ecology and Noospherology", "Soil Science". His e-mail address is VseVsevolodehotmail.com and his personal webpage at www.programmer.dp.ua/chernishenko.php. 
Hierarchical heterogenity of populations: modeling by the open Eigen hypercycle. V.Ye.Belozyorov, V.S. Chernyshenko, S.V. Chernyshenko. // Proceedings 26th European conference on modelling and simulation ECMS 2012, 2012, European council for modelling and simulation, Digitaldruck Pirrot GmbH, May 29 - June 01, pp. 150-156. 